\title{
Apolipoprotein A-IV as a novel gene associated with polycystic ovary syndrome
}

\author{
YONG-SOO KIM ${ }^{1 *}$, BON-HEE GU ${ }^{1 *}$, BUM-CHAE CHOI ${ }^{2,3^{*}}$, MYUNG-SUN KIM $^{1}$, SANGJIN SONG ${ }^{3}$, \\ JI-HYUN YUN ${ }^{1}$, MI KYUNG CHUNG ${ }^{1}$, CHEOL-HEE CHOI ${ }^{2}$ and KWANG-HYUN BAEK ${ }^{1}$ \\ ${ }^{1}$ Department of Biomedical Science, Fertility Center, CHA University, CHA General Hospital, Seoul; \\ ${ }^{2}$ Research Center for Resistant Cells, Department of Pharmacology, Chosun University Medical School, Gwangju; \\ ${ }^{3}$ Department of Obstetrics and Gynecology, CL Women's Hospital, Gwangju, Republic of Korea
}

Received September 30, 2012; Accepted November 26, 2012

DOI: $10.3892 /$ ijmm.2013.1250

\begin{abstract}
Polycystic ovary syndrome (PCOS) is a common endocrine-metabolic disorder, affecting 6-10\% of women of reproductive age. The etiology remains poorly understood. To investigate the differentially expressed proteins from PCOS patients versus healthy women, the protein expression in follicular fluid was analyzed using two-dimensional electrophoresis (2-DE). Since follicular fluid contains a number of secretory proteins required for oocyte fertilization and follicle maturation, it is possible that follicular fluid can be used as a provisional source for identifying pivotal proteins associated with PCOS. In this study, six overexpressed proteins [kininogen 1, cytokeratin 9, antithrombin, fibrinogen $\gamma$-chain, apolipoprotein A-IV (apoA-IV) precursor and $\alpha$-1-Bglycoprotein (A1BG)] in follicular fluids from PCOS patients were identified with matrix-assisted laser desorption/ionization-time-of-flight mass spectrometry (MALDI-TOF-MS) and nano LC-MS/MS. Western blot analysis confirmed that the protein expression levels of apoA-IV precursor and A1BG were increased in follicular fluid from PCOS patients compared with those from normal controls. The analysis of protein expression for other proteins revealed individual variation. These results facilitate the understanding of the molecular mechanisms of PCOS and provide candidate biomarkers for the development of diagnostic and therapeutic tools.
\end{abstract}

\section{Introduction}

Polycystic ovary syndrome (PCOS) is one of the most common endocrine disorders, affecting $6-10 \%$ of women of

Correspondence to: Professor Kwang-Hyun Baek, Department of Biomedical Science, CHA University, CHA General Hospital, 502 Yatap-Dong, Bundang-Gu, Seongnam-Si, Gyeonggi-Do 463-840, Republic of Korea

E-mail: baek@cha.ac.kr

${ }^{*}$ Contributed equally

Key words: apolipoprotein A-IV, follicular fluid, single nucleotide polymorphism, two-dimensional gel electrophoresis reproductive age, and is diagnosed by combination of polycystic ovaries, hyperandrogenism or oligo-anovulation (1-3). Serum luteinizing hormone ( $\mathrm{LH})$ hypersecretion, compensatory hyperinsulinemia from insulin resistance and reduced fecundity are common features of PCOS and at least $50 \%$ of PCOS women are insulin resistant when compared with age- and weight-matched controls (4). Genetic studies on this disorder have certain difficulties due to heterogeneity in the phenotype of PCOS patients. Therefore, association studies have been used as a powerful genetic approach to analyze the complex traits in PCOS. Most molecular and genetic analyses have focused on genes involved in folliculogenesis and insulin signaling (5-12).

Follicular fluid, secreted by granulose cells and by diffusion from the theca capillaries, plays an essential role in the physiology of follicular growth, oocyte maturation and ovulation (13). A number of studies have revealed that follicular fluid inhibits the binding of spermatozoa to the zona pellucida (14), and promotes hyperactivation and acrosome reaction of sperm $(15,16)$, and oocyte maturation and embryo development $(17,18)$. Moreover, follicular fluid contains high levels of gonadotropin and growth factors that are important to oocyte maturation and embryo development (19). Therefore, the chemical composition of fluid from dominant follicles can be used as an indicator of the secretory activities and metabolism of follicular cells, which regulate the follicular quality. Recent proteomic studies demonstrated that most proteins in human follicular fluid are also present in the plasma, suggesting that these proteins can be used as biomarkers for reproductive diseases using both follicular fluid and plasma (20-23). In this study, we performed genetic and proteomic analyses to identify novel PCOS-associated factors in follicular fluid from PCOS patients.

\section{Materials and methods}

Subjects and biochemical determinations. Women with PCOS were recruited at the Fertility Center at CHA General Hospital in Seoul, Korea. The criteria for diagnosis of PCOS rely on the combination of clinical symptoms, ultrasonographic examination, and biochemical data based on the revised diagnostic criteria announced in the 2003 ASRM/ESHRE Rotterdam 
Table I. Clinical characteristics of normal and PCOS patients for follicular fluid and blood serum.

\begin{tabular}{lcc}
\hline & Normal & PCOS \\
\hline No. & $5(3)$ & $5(7)$ \\
Body mass index $\left(\mathrm{kg} / \mathrm{m}^{2}\right)$ & $20.94(19.78-22.38)(20.02-21.36)$ & $23.07(19.97-26.35)[22.37(18.02-25.24)]$ \\
FSH $(\mathrm{IU} / \mathrm{l})$ & $6.1(3.7-8.2)[6.4(5.0-7.5)]$ & $5.74(2.9-8.2)[5.8(3.1-8.5)]$ \\
LH $(\mathrm{IU} / \mathrm{l})$ & $4.98(2.3-6.7)[2.49(1.5-4.0)]$ & $10.46(8.0-13.0)[7.28(0.4-24.1)]$ \\
$\mathrm{E}_{2}(\mathrm{pmol} / \mathrm{l})$ & $27.86(9.3-49.0)[17(11.76-25.13)]$ & $32.6(19.0-61.0)[27(7.81-52.66)]$ \\
\hline
\end{tabular}

Numbers in parentheses represent normal and polycystic ovary syndrome (PCOS) patients whose sera were used for western blot analysis.

consensus (24). Healthy female volunteers were enrolled and used as the control group. Their healthy state was determined based on medical history, physical and pelvic examination and complete blood chemistry. Their normal ovulatory state was confirmed by transvaginal ultrasonography and a plasma hormone assay was conducted during the luteal phase of the cycle.

The clinical characteristics of normal and PCOS patients are listed in Table I. Informed written consent was obtained from all participants. Follicular fluid was prepared as previously described (20). To carry out western blot analysis, serum samples were prepared as previously described (20). Venous blood samples were obtained prior to follicular aspiration, and were collected in sterile plastic tubes containing ethylenediaminetetraacetic acid (EDTA) as an anticoagulant and immediately centrifuged. All samples were stored at $-80^{\circ} \mathrm{C}$ until use.

For genetic analysis, 233 patients with PCOS and 120 healthy women of reproductive age were recruited from the Fertility Center at CHA General Hospital. Blood samples were obtained in tubes containing EDTA, which acts as an anticoagulant. The follicular fluid and blood serum used for proteomic analysis were also used in the genomic study. Genomic DNA was extracted using a DNA purification system kit (Gentra Systems, Minneapolis, MN, USA). Biochemical analysis of various hormones from blood including insulin, DHEA-S, testosterone, DHEA-S, FSH and LH was conducted. Furthermore, the participants underwent a series of physical examinations including weight, height and waist/hip ratio measurements, and the history of the patient and family were recorded (Table I). The human follicular fluid and blood samples obtained from controls and PCOS patients were approved by the CHA General Hospital Institutional Review Board.

Two-dimensional electrophoresis (2-DE) and image analysis. Immobiline DryStrips (IPG) pH 3-10 nonlinear $18 \mathrm{~cm}$, and Pharmalytes $\mathrm{pH}$ 3-10 were purchased from Amersham Pharmacia Biotech (Uppsala, Sweden); Griess reagent, CHAPS, urea, DTT, Tris base, thiourea, glycine, ammonium persulfate and SDS were obtained from Sigma (St. Louis, MO, USA); Coomassie brilliant blue (CBB) G-250 and TEMED were from Bio-Rad (Hercules, CA, USA). All other chemicals were obtained from various commercial sources and were of the highest grade.

Depletion of the six most abundant proteins (i.e., albumin, transferrin, $\operatorname{IgG}$, Ig A, haptoglobin and antitrypsin) in follicular fluid was carried out using a Multiple Affinity Removal Column (MARC) (Agilent Technologies, Inc., Wilmington, DE, USA). Then, 2-DE and image analyses were performed, as previously described (20).

In-gel digestion and LC-MS/MS. Excised spots were destained, reduced, alkylated, and then digested with trypsin (Promega, Madison, WI, USA) as previously described (25). For matrix-assisted laser desorption/ionization-time-of-flight mass spectrometry (MALDI-TOF-MS) analysis, the tryptic peptides were concentrated using a poros R2 and oligo R3 column (Applied Biosystems, Foster City, CA, USA) and eluted in $\alpha$-cyano-4-hydroxycinnamic acid. Spectra were obtained using a 4700 TOF/TOF spectrophotometer (Applied Biosystems). Proteins were identified from the peptide mass maps using MASCOT (http://www.matrixscience.com/search form_select.html), MS-Fit (http://prospector.ucsf.edu), and ProFound (http://prowl.rockfeller.edu/prowl-cgi/profound.exe) to search for the proteins in the protein database, Swiss-Prot and GenBank. For nano LC-ESI-MS/MS analysis, the peptides digested with trypsin were concentrated using a poros R2 and oligo R3 column (Applied Biosystems).

Bioinformatics. Each MS/MS spectrum obtained was searched against the non-redundant protein sequence database using the Spectrum Mill software tool. Sequences of uninterpreted CID spectra were identified by correlation with the peptide sequences present in the protein sequence database (NCBInr 2012.1) using the Spectrum Mill MS Proteomics Workbench (Rev A.03.00.015; Agilent Technologies, Inc.).

Western blot analysis. Human follicular fluid proteins diluted to $1 / 10$ with PBS buffer and human sera were subjected to SDS-PAGE. Albumin was removed from the follicular fluid to detect kininogen 1, cytokeratin 9, antithrombin, fibrinogen $\gamma$-chain, apolipoprotein A-IV (apoA-IV) precursor, and $\alpha$-1-Bglycoprotein (A1BG) using the Aurum serum protein mini kit (Bio-Rad). The proteins were loaded in each lane of a 7.5\% SDS-PAGE and blotted onto nitrocellulose membranes. The membranes were incubated with a blocking solution, containing a 1:200 dilution of anti-kininogen 1 (Santa Cruz Biotechnology, Inc., Santa Cruz, CA, USA), or anti-cytokeratin 9 antibodies (Abcam, Cambridge, UK). In addition, an antithrombin antibody (Abcam) diluted to 1:1,000 with the blocking solution was used. Also, the membranes were incubated with a blocking solution, containing a 1:500 dilution of 


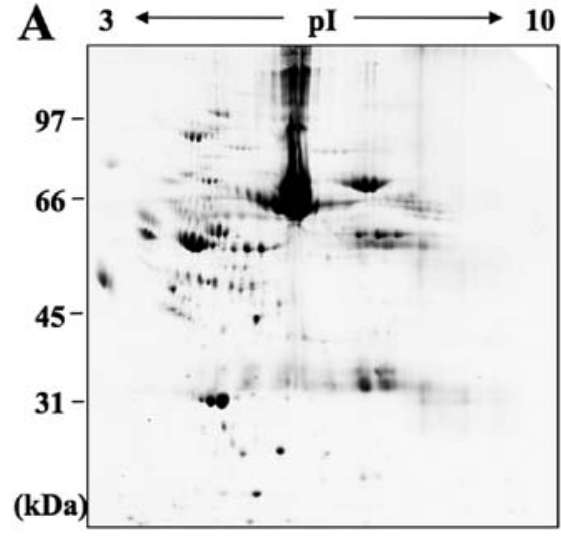

before depletion with MARC

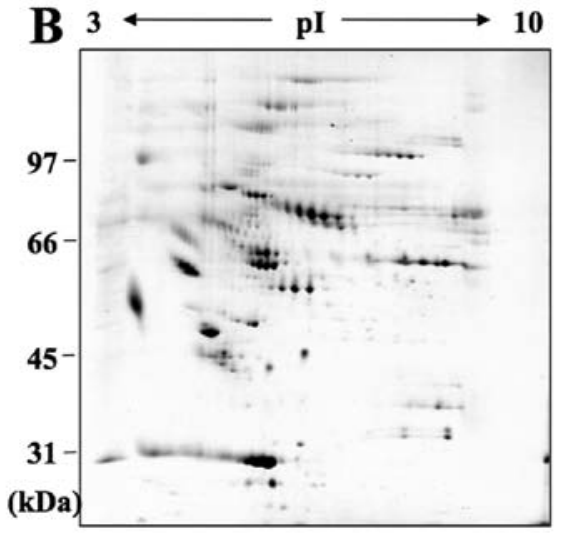

after depletion with MARC

Figure 1.2-DE images of follicular fluid. The most abundant proteins in follicular fluid derived from a normal patient were depleted using an immunoaffinity column (MARC) and then separated by 2-DE. 2-DE images of follicular fluid (A) before, and (B) after depletion.

anti-fibrinogen $\lambda$, anti-apoA-IV (Santa Cruz Biotechnology, Inc.), or anti-A1BG (Aviva Systems Biology, San Diego, CA, USA) antibodies. The membranes were then incubated with a blocking solution containing a 1:5,000 dilution of horseradish peroxidase-conjugated secondary antibodies (KPL, Gaithersburg, MD, USA). An ECL system (Elpis Biotech, Daejeon, Korea) was used to detect signals. Bands from the western blot analysis were scanned and digitized using Fluor-S $S^{\text {TM }}$ MultiImager (Bio-Rad). Values are expressed as the means \pm standard error of the mean (SEM).

Genetic analysis. Restriction fragment length polymorphism (RFLP) analysis was performed for apoA- $I V$ using isolated genomic DNA from the blood samples obtained from all subjects. PCR primers were designed and used to amplify the DNA for the genotyping analysis. To determine the Gln360His variant genotype, the following primers were designed: forward, 5'-CTT CCA GAT GAA GAA GAA CG-3' and reverse, 5'-AGT TTC TGC CTG AGC TGT T-3'. The PCR conditions were as follows: denaturation at $94^{\circ} \mathrm{C}$ for $30 \mathrm{sec}$, annealing at $55^{\circ} \mathrm{C}$ for $10 \mathrm{sec}$ and polymerization at $72^{\circ} \mathrm{C}$ for $15 \mathrm{sec}$. Amplified PCR products were purified using a PCR purification kit (AccuPrep ${ }^{\circledR}$; Bioneer, Daejeon, Korea) and screened by restriction digestion with Fnu4HI (New England Biolabs, Beverly, MA, USA) at $37^{\circ} \mathrm{C}$ for $6 \mathrm{~h}$. In addition, amplified PCR products for each genotype were sequenced in order to confirm the genotyping results from the RFLP analysis.

Statistical analysis. For the statistical analysis, the HapAnalyzer (National Genome Research Institute, Seoul, Korea) and SAS ver. 9.1 (2006) were used to analyze the distributions of the apoA-IV genotype. $\mathrm{P}$-value $<0.05$ was considered to indicate a statistically significant difference.

\section{Results}

2-DE analysis on follicular fluid of PCOS. A typical 2-DE separation was performed in the $\mathrm{pH}$ range of 3-10 on proteins extracted from the follicular fluid of PCOS patients and healthy women as a control to identify proteins that were aberrantly expressed in PCOS patients. In order to deplete the samples of the most abundant proteins found in follicular fluid, an immunoaffinity column (MARC) was applied to collect flow-through fractions (Fig. 1), as previously described (20).

Follicular fluids were collected from in vitro fertilization (IVF) patients suffering from PCOS, and from normal controls who underwent an IVF cycle due to male infertility factors with the treatment of the GnRH agonist for pituitary downregulation. Clinical and biochemical profiles of PCOS patient and normal control groups are shown in Table I. To ascertain differences in protein expression between normal and PCOS patients, we applied the isoelectric focusing technique using differential-display proteomic analysis methods described previously (20). Follicular fluid depleted of the most abundant proteins with an immunoaffinity column revealed better distinguishable spots on 2-DE gels (Fig. 1). The same separation method was then performed on proteins extracted from the follicular fluid of PCOS patients and healthy women as a control to identify proteins that were aberrantly expressed in PCOS patients (Fig. 2A and B).

Identification of differentially expressed proteins in PCOS patients. When protein expression levels in the follicular fluid from five PCOS patients with polycystic ovaries, oligo- or amenorrhea and hyperandrogenism, were compared with five normal women as a control, six spots were determined to be overexpressed in the 2-DE analysis based on relative fold increase in volume intensity (Fig. 2C-E). The expression level was determined by examining the ratio of the relative spot volume of a protein in the gel (Fig. 2F). The identification of these different proteins was performed by MALDI-TOF-MS or nano LC-MS/MS. The identified proteins included kininogen 1 , cytokeratin 9, antithrombin chain $\mathrm{B}$, fibrinogen $\gamma$-chain, apoA-IV precursor and A1BG (Table II).

Western blot analysis using follicular fluid and plasma. The follicular fluids were analyzed by western blot analysis using the appropriate antibodies to validate their identities. As shown in Fig. 3A, apoA-IV was detected in the follicular fluid of both the PCOS and the control group. ApoA-IV 


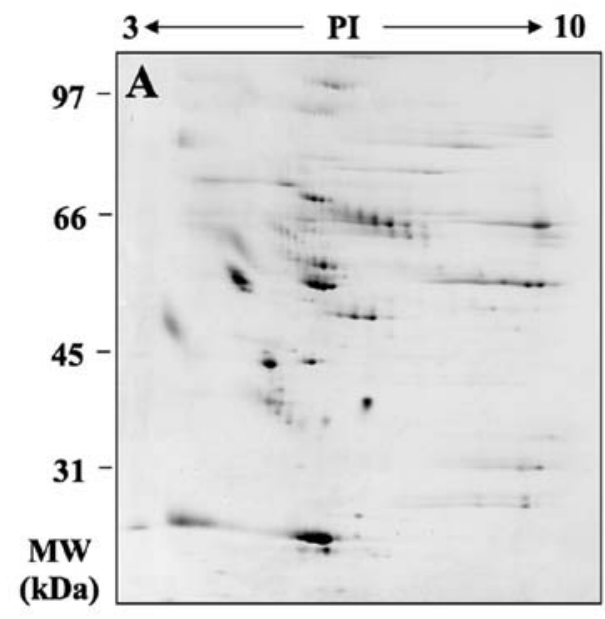

Control

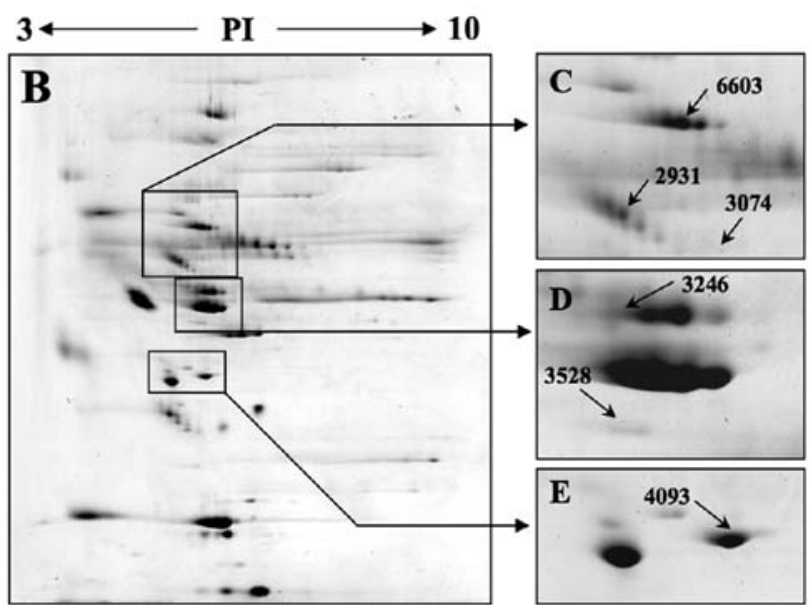

$\operatorname{PCOS}$

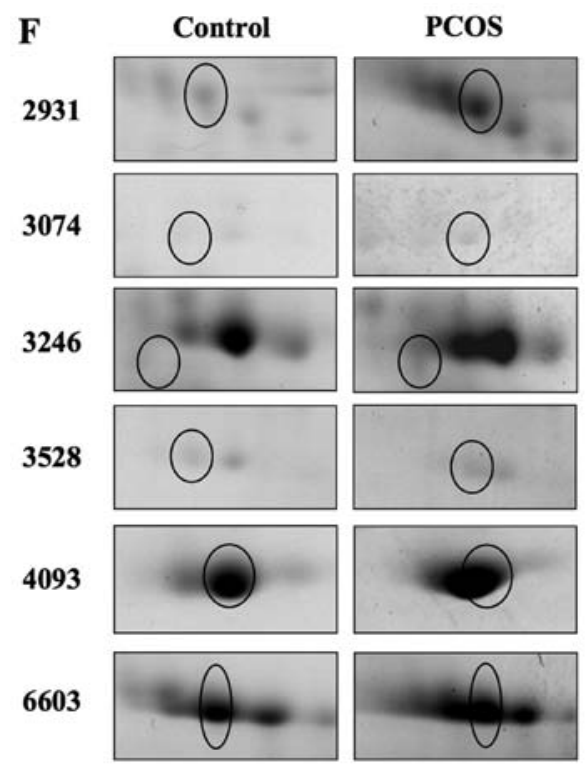

Figure 2. Master images of 2-DE stained with Coomassie blue G-250. One milligram follicular fluid proteins were prepared as described in Materials and methods and separated on an $18 \mathrm{~cm} \mathrm{pH} \mathrm{3-10} \mathrm{IPG} \mathrm{strip} \mathrm{and} \mathrm{then} \mathrm{a} \mathrm{9-16 \%} \mathrm{gradient} \mathrm{SDS-PAGE.} \mathrm{(A)} \mathrm{2-DE} \mathrm{image} \mathrm{of} \mathrm{follicular} \mathrm{fluid} \mathrm{from} \mathrm{a} \mathrm{control} \mathrm{(healthy}$ woman), (B) 2-DE image of follicular fluid from a PCOS patient. (C-E) In the follicular fluid from a PCOS patient, 6 spots were determined to be overexpressed in 2-DE analysis based on the relative fold increase in volume intensity. (F) Proteins identified by MALDI-TOF-MS: 2931, kininogen 1; 3074, cytokeratin 9; 3246, chain B, antithrombin; 3528, fibrinogen $\gamma$-chain; 4093, apolipoprotein A-IV precursor; and 6603, $\alpha$-1-B-glycoprotein.

(45 kDa) expression in PCOS patients was significantly higher than that in controls (1.00 vs. 3.21 $00.89, \mathrm{P}<0.005)$ (Fig. 3B). Fig. 3C shows the western blot analysis of the A1BG $(52 \mathrm{kDa})$, which was detected in the follicular fluid of controls and PCOS patients. A1BG expression in PCOS patients was also increased by $\sim 3$-fold compared with the control (1.00 vs. $2.70 \pm 0.31, \mathrm{P}<0.005$ ) (Fig. 3D). On the other hand, western blot analysis revealed individual variations in the expression of kininogen 1, cytokeratin 9, fibrinogen $\gamma$ and antithrombin between the PCOS and the control group (Fig. 4A). Fig. 4A shows that the expression level of these proteins varied among normal or PCOS patients.

Since the plasma protein enrichment process occurs during the production of follicular fluid, western blot analysis was conducted using human sera derived from the control and PCOS patients (Fig. 4B). Clinical and biochemical profiles of PCOS patient and normal control groups are shown in paren- theses in Table I. In human sera from PCOS patients, apoA-IV, A1BG, antithrombin and fibrinogen $\gamma$ were not significantly altered in their expression levels (Fig. 4B). However, cytokeratin 9, kininogen 1 and actin were not detected at the protein level (data not shown).

Association study between apoA-IV and PCOS using RFLP analysis. Several researchers have performed association studies between various genes and PCOS for genetic analysis to identify the etiology of PCOS. Therefore, in this report, an association study for apoA- $I V$, which was highly expressed at the protein level in follicular fluid and sera of PCOS patients, was also conducted. RFLP analysis was performed on the samples collected from 233 PCOS and 120 control patients. All PCOS patients had clinical and biochemical characteristics in accordance with the definition by ASRM/ESHRE (24); 149 patients $(63.95 \%)$ showed polycystic ovaries and oligo- or 


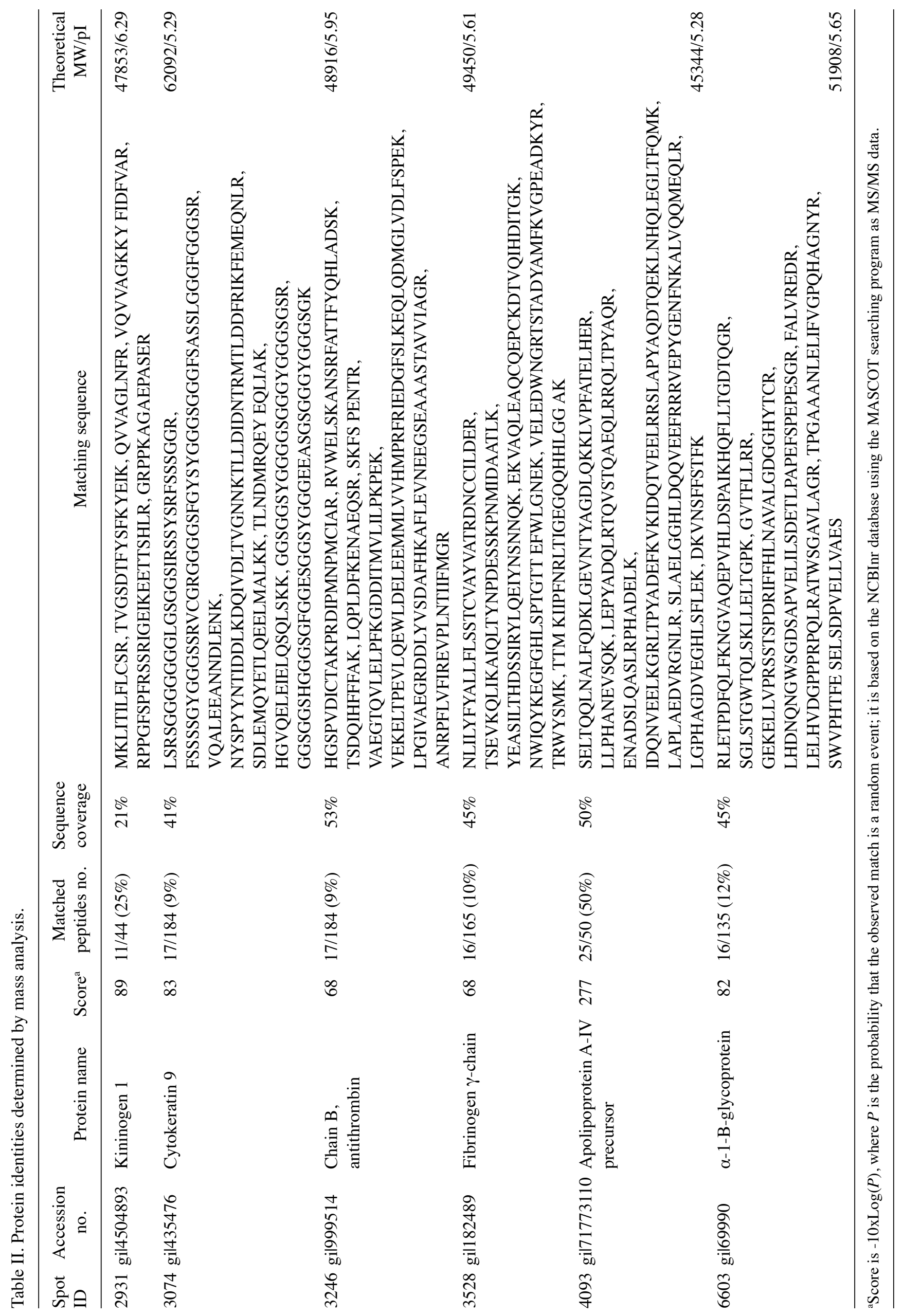


A

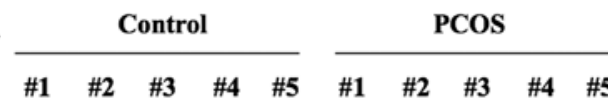

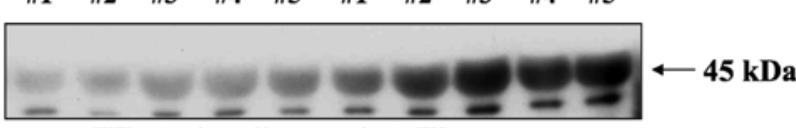

WB: Anti-apolipoprotein A-IV precursor

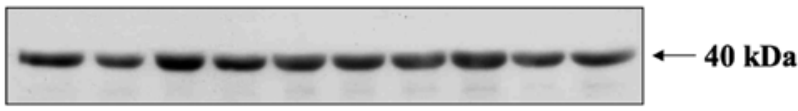

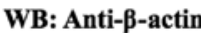

B

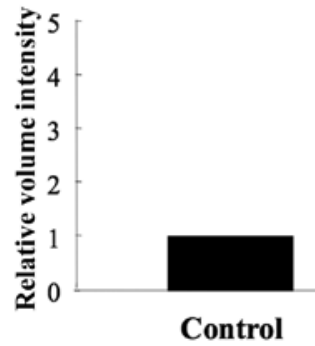

Figure 3. Western blot analysis of apolipoprotein A-IV precursor. Western blot analysis of the follicular fluid proteins from controls and PCOS patients was carried out. (A) Western blot analysis using an anti-apolipoprotein antibody. (B) Quantitative density of gel bands for apolipoprotein A-IV (45 kDa). (C) Western blot analysis using anti- $\alpha-1$-glycoprotein antibody. (D) Quantitative density of gel bands in the $\alpha$-1-B-glycoprotein (52 kDa). The bars represent the mean \pm SEM of the density of the gel bands determined from PCOS patients and controls. Significant difference was assessed by a Student's $t$-test ("P $<0.005)$.
C

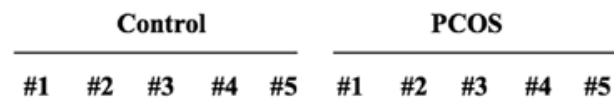

D

莺

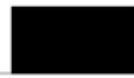

Control

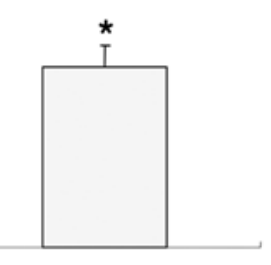

PCOS
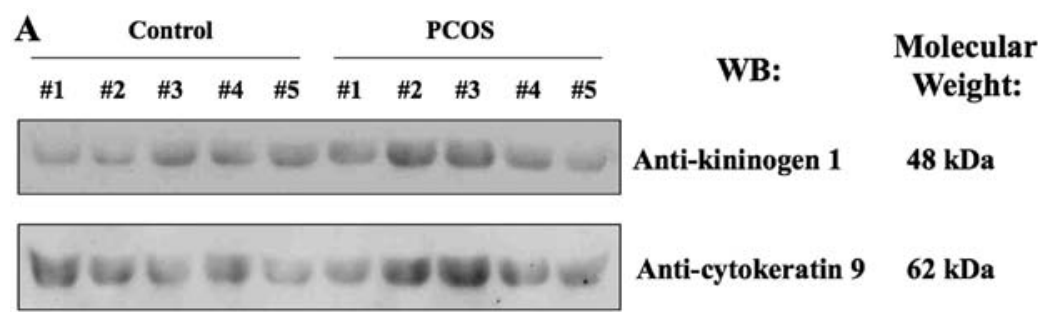

Anti-cytokeratin $9 \quad 62 \mathrm{kDa}$

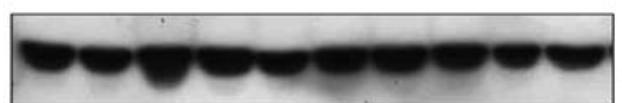

Anti-antithrombin

$48 \mathrm{kDa}$

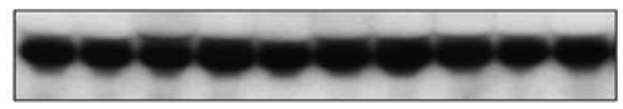

Anti-fibrinogen $\gamma$

$49 \mathrm{kDa}$

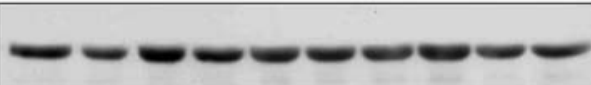

Anti- $\beta$-actin

$40 \mathrm{kDa}$

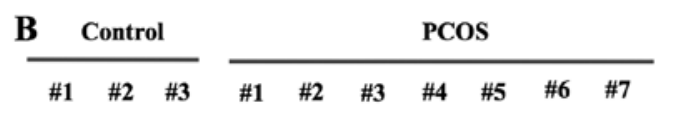

WB:

\section{Molecular}

Weight:

Anti-apolipoprotein A-IV 45 kDa

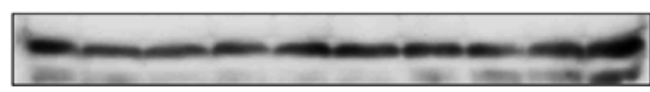

Anti-a-1-B-glycoprotein

$52 \mathrm{kDa}$

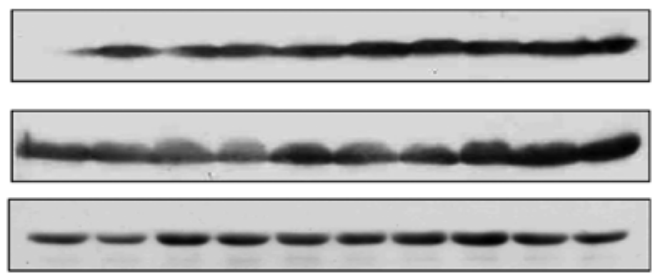

Anti-antithrombin

$48 \mathrm{kDa}$

Anti-fibrinogen $\gamma$

\section{$47 \mathrm{kDa}$}

Anti- $\beta$-actin

$40 \mathrm{kDa}$

Figure 4. (A) Western blot analysis of kininogen 1 (48 kDa), cytokeratin 9 (62 kDa), antithrombin (48 kDa), and fibrinogen $\gamma(49 \mathrm{kDa})$ in the follicular fluid of PCOS patients and normal controls. Individual variation of protein expression is shown. (B) Western blot analysis of apolipoprotein A-IV (45 kDa), $\alpha-1-\mathrm{B}$ glycoprotein $(52 \mathrm{kDa})$, antithrombin $(48 \mathrm{kDa})$, and fibrinogen $\gamma(47 \mathrm{kDa})$ in human sera of PCOS patients and normal controls. Individual variation of protein expression is shown. 
Table III. Clinical and biochemical characteristics of normal controls $(n=120)$ and patients with PCOS $(n=233)$.

\begin{tabular}{lcc}
\hline Characteristics & Normal controls $(\mathrm{n}=120)$ & PCOS patients $(\mathrm{n}=233)$ \\
\hline Age $($ year) & $33.13 \pm 3.36(29-36)$ & $32.41 \pm 3.04(29-35)$ \\
BMI $\left(\mathrm{kg} / \mathrm{m}^{2}\right)$ & $20.88 \pm 2.62$ & $22.78 \pm 3.76$ \\
Waist/hip ratio (WHR) & $0.80 \pm 0.05$ & $0.82 \pm 0.06$ \\
Obesity & $\mathrm{n}=6(5.45 \%)$ & $\mathrm{n}=44(18.88 \%)$ \\
Polycystic ovaries and oligo- or amenorrhea & $\mathrm{n}=0(0.00 \%)$ & $\mathrm{n}=149(63.95 \%)$ \\
Polycystic ovaries and hyperandrogenism & $\mathrm{n}=0(0.00 \%)$ & $\mathrm{n}=27(11.59 \%)$ \\
Oligo- or amenorrhea and hyperandrogenism & $\mathrm{n}=0(0.00 \%)$ & $\mathrm{n}=31(13.30 \%)$ \\
Polycystic ovaries, oligo- or amenorrhea, and & $\mathrm{n}=0(0.00 \%)$ & $\mathrm{n}=26(11.16 \%)$ \\
hyperandrogenism & & \\
FSH levels $(\mathrm{mlU} / \mathrm{ml})$ & $6.13 \pm 2.23(3.20-8.49)$ & $6.61 \pm 7.17(3.00-11.50)$ \\
LH levels $(\mathrm{mlU} / \mathrm{ml})^{\mathrm{a}}$ & $3.32 \pm 3.06(1.00-17.02)$ & $6.63 \pm 4.78(1.00-7.10)$ \\
E levels $(\mathrm{pg} / \mathrm{ml})$ & $71.49 \pm 298.60(9.10-81.40)$ & $52.92 \pm 128.92(5.00-219.90)$ \\
Prolactin levels $(\mathrm{ng} / \mathrm{ml})$ & $13.79 \pm 10.47(2.30-20.90)$ & $14.59 \pm 13.44(4.10-46.40)$ \\
TSH levels $(\mu \mathrm{lU} / \mathrm{ml})$ & $2.98 \pm 6.57(0.46-5.47)$ & $2.59 \pm 5.08(0.03-4.06)$ \\
DHEA-S levels $(\mu \mathrm{g} / \mathrm{dl})^{\mathrm{a}}$ & $168.10 \pm 70.35(45.30-377.20)$ & $194.86 \pm 77.93(67.20-257.40)$ \\
Testosterone $(\mathrm{ng} / \mathrm{ml})^{\mathrm{a}}$ & $0.23 \pm 0.14(0.06-0.86)$ & $0.38 \pm 0.22(0.01-0.54)$ \\
\hline
\end{tabular}

andicates P-value <0.05 statistically. PCOS, polycystic ovary syndrome.

Table IV. Allele frequencies of the Gln360His ApoA-IV gene.

\begin{tabular}{lcc}
\hline & PCOS patient group & Control group \\
\hline Genotype & & $117(97.5 \%)$ \\
GG & $138(59.2 \%)$ & $3(2.5 \%)$ \\
GT & $45(19.3 \%)$ & $0(0.0 \%)$ \\
TT & $50(21.5 \%)$ \\
Co-dominant & OR $(95 \% \mathrm{CI})=15.68(5.14-47.89)$, P-value $<0.001$ \\
Dominant & OR $(95 \% \mathrm{CI})=28.71(8.83-93.33)$, P-value $<0.001$ \\
Recessive & OR $(95 \% \mathrm{CI})=\mathrm{N} / \mathrm{A}, \mathrm{P}-\mathrm{value}=0.997$ \\
Total & OR $(95 \% \mathrm{CI})=37.33(11.74-118.70)$, P-value $<0.001$
\end{tabular}

PCOS, polycystic ovary syndrome.

amenorrhea, 31 patients $(13.3 \%)$ had oligo- or amenorrhea and hyperandrogenism, 27 patients $(11.59 \%)$ had polycystic ovaries and hyperandrogenism, and 26 patients $(11.16 \%)$ exhibited polycystic ovaries, oligo- or amenorrhea, and hyperandrogenism. The level of LH, DHEA-S and testosterone were significantly high in the PCOS group (Table III).

In the RFLP analysis, the association of apoA-IV and PCOS was confirmed by the $\mathrm{G} \rightarrow \mathrm{T}$ substitution, which led to a change from glutamine to histidine, by restriction enzyme Fnu4HI (Fig. 5). While restriction enzyme Fnu4HI cleaved normal DNA fragments into 101 and 26 bp (Fig. 5A), variant DNA fragments by the $\mathrm{G} \rightarrow \mathrm{T}$ substitution were not cleaved (Fig. 5B).

The homozygote of the variant allele (TT) was not detected in the healthy control group and heterozygote genotype (GT) was only observed in three control patients. Of note, 50 PCOS patients $(21.5 \%)$ had the TT variant homozygote genotype, and 45 PCOS patients $(19.3 \%)$ had the GT variant heterozygote genotype (Table IV). Statistical analysis using HapAnalyzer and SAS programs identified that the Gln360His variant was significantly associated with PCOS in dominant $(\mathrm{P}<0.05)$ and co-dominant $(\mathrm{P}<0.05)$ (Table IV). However, the genotype of PCOS patients used for proteomic analysis only displayed the GG homozygote.

\section{Discussion}

PCOS is a common endocrinopathy affecting 6-10\% of women of reproductive age (1). There is clear evidence of the underlying genetic cause for PCOS based on familiar clustering of cases. Most studies have been consistent with an autosomal dominant pattern of inheritance. However, it has been difficult to draw solid conclusions from several of these studies due to the small sample sizes, errors in statistical analysis, and differences in diagnostic criteria, an inevitable consequence of PCOS being 


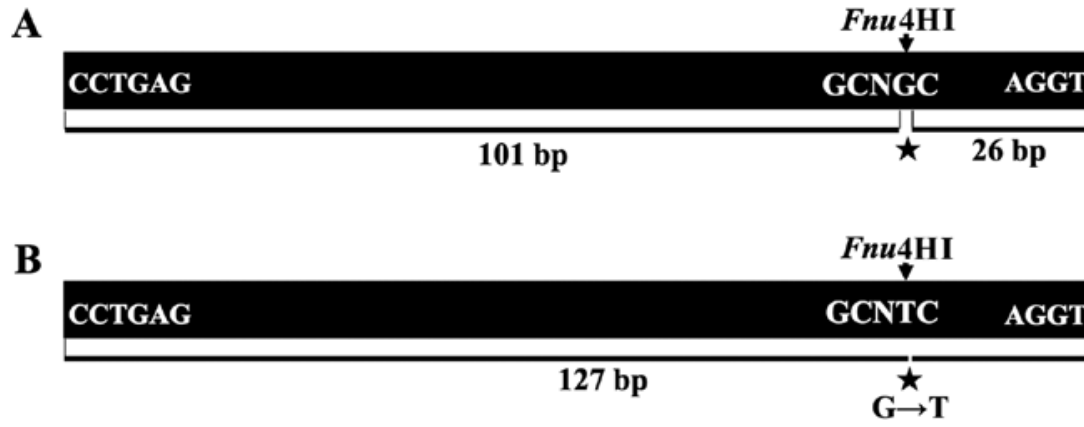

101 bp and 26 bp: Homozygosity for $G$ allele

127 bp: Homozygosity for $T$ allele

127 bp, 101 bp and 26 bp: Heterozygosity for $G$ and $T$ alleles

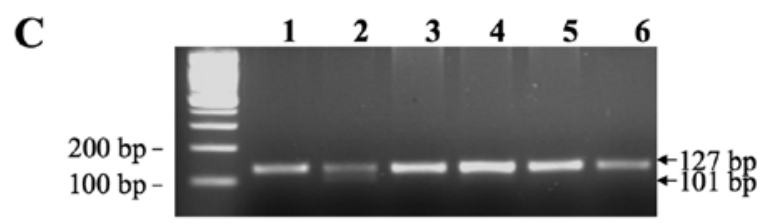

\section{Lane 1: GG Lane 2: GT Lane 3: GG \\ Lane 4: GG Lane 5: GG Lane 6: GG}

Figure 5. Structure of the apoA-IV gene. (A) The $127 \mathrm{bp}$ fragment was amplified by PCR. The major allele is the G allele, which is recognized by the restriction enzyme Fnu4HI. (B) If the G allele was modified to the T allele, then the restriction enzyme Fnu4HI does not recognize the site. (C) The G/T polymorphism in the apoA-IV gene (Gln360His) by RFLP analysis.

a heterogeneous disorder. It has been suggested that PCOS develops as a consequence of a primary genetic abnormality in ovarian androgen production, interacting with environmental factors or other factors causing hyperinsulinemia.

Body fluids such as follicular fluid, amniotic fluid and cervical-vaginal fluid have been used to investigate the pathogenesis for pregnancy and birth including recurrent pregnancy loss (RPL), preeclampsia, intra-amniotic infection (20-23,25,26). In order to investigate novel etiology factors of PCOS, the proteins expressed in the follicular fluid of PCOS patients were investigated using 2-DE analysis, and six proteins were found to be aberrantly expressed. These proteins were kininogen 1 , cytokeratin 9, antithrombin chain B, fibrinogen $\gamma$, apoA-IV precursor and A1BG (Fig. 3). However, it is unclear if these proteins were induced due to the ovarian hyperstimulation caused by IVF therapy even though control samples did not show upregulation of these proteins.

ApoA-IV is a $45 \mathrm{kDa}$ glycoprotein triglyceride-rich glycoprotein (27). It is expressed in the small intestine and its synthesis and secretion increases in response to fatty meals (28). ApoA-IV has several proposed roles, including lipid transport, lipoprotein metabolism (29), control of food intake (30) and gastric function (31). apoA-IV has been shown to be antiatherogenic (32), and there is also evidence supporting apoA-IV antioxidant activity (32-34). Two mechanisms have been suggested for apoA-IV's antiatherogenic action: enhancement of cellular lipid efflux (i.e., 'reverse cholesterol transport') (35) and antioxidant activity $(33,34)$.
We showed that the expression of apoA-IV was significantly increased in the follicular fluid from PCOS patients. One of the possibilities is that this may result in aberration of lipoprotein metabolism and transport, causing the generation of polycystic ovaries. Identifying the etiology of PCOS is extremely difficult as it is caused by a combination of hormonal, genetic and environmental factors. For these reasons, a combination of proteomic and genetic analyses has been used to identify new factors associated with these diseases (35-37). Several researchers have investigated a diverse range of genes that may be associated with PCOS to identify the etiology of this disease, and apoA-IV has also been studied in numerous diseases, including obesity, using genetic analysis (38). Gln360His and Thr347Ser are well-studied functional polymorphisms of apoA-IV. It has been demonstrated that these affect obesity and lipid/lipoprotein metabolism $(39,40)$. In particular, Gln360His polymorphism is related with type 1 diabetes mellitus (T1DM) and obesity $(41,42)$.

In women with PCOS, insulin resistance or deficiency is one of the main characteristics of patients with obesity $(43,44)$. Therefore, we performed a single nucleotide polymorphism (SNP) study for Gln360His polymorphism of apoA-IV to investigate its association with PCOS. In these experiments, this polymorphism was shown to be highly related to PCOS. The homozygote of the variant allele was not detected in the control group. This suggests that the polymorphism affects the development of PCOS by regulating apoA-IV protein expression or by impacting the function of the protein. However, the 
relationship between the aberrant expression of apoA-IV in follicular fluid and Gln360His polymorphism and their role in the generation of PCOS remains unclear. The study had certain challenges due to the fact that PCOS is an endocrine disorder influenced by diverse factors.

We also determined the expression level of $\alpha-1-B$ glycoprotein (A1BG) by western blot analysis, and found that the expression of this protein is increased in PCOS patients relative to healthy women. A1BG is a known plasma protein with unknown function and a member of the immunoglobulin superfamily. Little is known about the biological functions of A1BG with regard to infertility diseases. Therefore, further studies are needed to explain why the A1BG protein is increased in PCOS patients.

Although we showed that the expression of kininogen 1 and cytokeratin 9 was increased in the follicular fluids from PCOS patients by 2-DE analysis, western blot analysis showed individual variation between PCOS and normal patients. However, the expression level also varied among normal or PCOS patients, suggesting that these proteins may be involved in the etiology of PCOS in certain cases. Therefore, continuous investigation with 2-DE analysis is required for the identification of proteins associated with the pathogenesis of PCOS.

In addition, we showed that the expression of antithrombin and fibrinogen $\gamma$ was increased in the follicular fluids from PCOS patients by 2-DE analysis. Western blot analysis, however, showed individual variation between PCOS and normal patients. In the case of antithrombin, Tsanadis et al (45) reported that the concentration difference was not statistically significant between women with PCOS and the control women, which is in agreement with our data. There are several studies in the literature regarding the concentration of fibrinogen in females with PCOS. Atiomo et al (46) reported a statistically higher concentration of fibrinogen in women with PCOS than in normal control women. Dahlgren et al (47) found that the concentration of fibrinogen was lower in females with PCOS. Kelly et al (48) did not find statistically significant differences between these two groups. Considering the discrepancies in the literature with regard to the expression level of fibrinogen in PCOS patients, further studies are required in order to more accurately understand the function of fibrinogen in PCOS patients.

\section{Acknowledgements}

This study was supported by a grant of the Korea Health 21 R\&D Project, Ministry of Health and Welfare, Republic of Korea (01-PJ10-PG6-01GN13-0002) and the Biomedical Proteome Research Center (A030003).

\section{References}

1. Goodarzi MO, Dumesic DA, Chazenbalk G and Azziz R: Polycystic ovary syndrome: etiology, pathogenesis and diagnosis. Nat Rew Endocrinol 7: 219-231, 2011.

2. Hart R, Hickey M and Franks S: Definitions, prevalence and symptoms of polycystic ovaries and polycystic ovary syndrome. Best Pract Res Clin Obstet Gynaecol 18: 671-683, 2004.

3. Pasquali R, Stener-Victorin E, Yildiz BO, Duleba AJ, Hoeger K, Mason H, Homburg R, Hickey T, Franks S, Tapanainen JS, et al: PCOS Forum: research in polycystic ovary syndrome today and tomorrow. Clin Endocrinol (Oxf) 74: 424-433, 2011.
4. Dumesic DA, Abbott DH and Padmanabhan V: Polycystic ovary syndrome and its developmental origins. Rev Endocr Metab Disord 8: 127-141, 2007.

5. Hourvitz A, Kuwahara A, Hennebold JD, Tavares AB, Negishi H, Lee TH, Erickson GF and Adashi EY: The regulated expression of the pregnancy-associated plasma protein-A in the rodent ovary: a proposed role in the development of dominant follicles and of corpora lutea. Endocrinology 143: 1833-1844, 2002.

6. Teixeira Filho FL, Baracat EC, Lee TH, Suh CS, Matsui M, Chang RJ, Shimasaki S and Erickson GF: Aberrant expression of growth differentiation factor-9 in oocytes of women with polycystic ovary syndrome. J Clin Endocrinol Metab 87: 1337-1344, 2002.

7. Munir I, Yen HW, Baruth T, Tarkowki R, Azziz R, Magoffin DA and Jakimiuk AJ: Resistin stimulation of 17alpha-hydroxylase activity in ovarian theca cells in vitro: relevance to polycystic ovary syndrome. J Clin Endocrinol Metab 90: 4852-4857, 2005.

8. Wickenheisser JK, Nelson-Degrave VL and McAllister JM: Dysregulation of cytochrome P450 17alpha-hydroxylase messenger ribonucleic acid stability in theca cells isolated from women with polycystic ovary syndrome. J Clin Endocrinol Metab 90: 1720-1727, 2005.

9. Goodarzi MO, Jones MR, Chen YD and Azziz R: First evidence of genetic association between AKT2 and polycystic ovary syndrome. Diabetes Care 31: 2284-2287, 2008.

10. Kim JJ, Choi YM, Hong MA, Hwang SS, Yoon SH, Chae SJ, Jee BC, Ku SY, Kim JG and Moon SY: Phosphatidylinositol 3-kinase p85alpha regulatory subunit gene Met326Ile polymorphism in women with polycystic ovary syndrome. Hum Reprod 24: 1184-1190, 2009.

11. Gu BH and Baek KH: Pro12Ala and His447His polymorphisms of PPAR-gamma are associated with polycystic ovary syndrome. Reprod Biomed Online 18: 644-650, 2009.

12. Choi SW, Gu BH, Ramakrishna S, Park JM and Baek KH: Association between a single nucleotide polymorphism in MTHFR gene and polycystic ovary syndrome. Eur J Obstet Gynecol Reprod Biol 145: 85-88, 2009.

13. Fortune JE: Ovarian follicular growth and development in mammals. Biol Reprod 50: 225-232, 1994.

14. Chiu PC, Koistinen R, Koistinen H, Seppala M, Lee KF and Yeung WS: Zona-binding inhibitory factor-1 from human follicular fluid is an isoform of glycodelin. Biol Reprod 69: 365-372, 2003.

15. Coy P, Gadea J, Romar R, Matas C and Garcia E: Effect of in vitro fertilization medium on the acrosome reaction, cortical reaction, zona pellucida hardening and in vitro development in pigs. Reproduction 124: 279-288, 2002.

16. Yao Y, Ho P and Yeung WS: Effects of human follicular fluid on the capacitation and motility of human spermatozoa. Fertil Steril 73: 680-686, 2002.

17. Wang TH, Chang CL, Wu HM, Chiu YM, Chen CK and Wang HS: Insulin-like growth factor-II (IGF-II), IGF-binding protein-3 (IGFBP-3), and IGFBP-4 in follicular fluid are associated with oocyte maturation and embryo development. Fertil Steril 86: 1392-1401, 2006

18. Wu YT, Tang L, Cai J,Lu XE, Xu J,Zhu XM, Luo Q and Huang HF: High bone morphogenetic protein-15 level in follicular fluid is associated with high quality oocyte and subsequent embryonic development. Hum Reprod 22: 1526-1531, 2007.

19. Rosen MP, Zamah AM, Shen S, Dobson AT, McCulloch CE, Rinaudo PF, Lamb JD and Cedars MI: The effect of follicular fluid hormones on oocyte recovery after ovarian stimulation: FSH level predicts oocyte recovery. Reprod Biol Endocrinol 7: 35, 2009.

20. Kim YS, Kim MS, Lee SH, Choi BC, Lim JM, Cha KY and Baek KH: Proteomic analysis of recurrent pregnancy loss: Identification of an inadequately expressed set of proteins in human follicular fluid. Proteomics 6: 3445-3454, 2006.

21. Kim MS, Gu BH, Song S, Choi BC, Cha DH and Baek KH: ITI-H4, as a biomarker in the serum of recurrent pregnancy loss (RPL) patients. Mol Biosyst 7: 1430-1440, 2011.

22. Atiomo W, Khalid S, Parameshweran S, Houda M and Layfield R: Proteomic biomarkers for the diagnosis and risk stratification of polycystic ovary syndrome: a systematic review. BJOG 116: 137-143, 2009.

23. Jarkovska K, Martinkova J, Liskova L, Halada P, Moos J, Rezabek K, Gadher SJ and Kovarova H: Proteome mining of human follicular fluid reveals a crucial role of complement cascade and key biological pathways in women undergoing in vitro fertilization. J Proteome Res 9: 1289-1301, 2010.

24. Rotterdam ESHRE/ASRM-Sponsored PCOS consensus workshop group: Revised 2003 consensus on diagnostic criteria and long health risks related to polycystic ovary syndrome (PCOS). Hum Reprod 19: 41-47, 2004. 
25. Gravett MG, Thomas A, Schneider KA, Reddy AP, Dasari S, Jacob T, Lu X, Rodland M, Pereira L, Sadowsky DW, et al: Proteomic analysis of cervical vaginal fluid: identification of novel biomarkers for detection of intra-amniotic infection. J Proteome Res 6: 89-96, 2007.

26. Park JS, Oh KJ, Norwitz ER, Han JS, Choi HJ, Seong HS, Kang YD, Park CW, Kim BJ, Jun JK, et al: Identification of proteomic biomarkers of preeclampsia in amniotic fluid using SELDI-TOF mass spectrometry. Reprod Sci 15: 457-468, 2008.

27. Sadeghi M, Roohafza H, Afshar H, Rajabi F, Ramzani M, Shemirani H and Sarafzadeghan N: Relationship between depression and apolipoproteins A and B: a case-control study. Clinics (Sao Paulo) 66: 113-117, 2011.

28. Ferrer F, Nazih H, Zair Y, Krempf M and Bard JM: Postprandial changes in the distribution of apolipoprotein AIV between apolipoprotein B- and non apolipoprotein B-containing lipoproteins in obese women. Metabolism 52: 1537-1541, 2003.

29. Araki S, Okazaki M and Goto S: Impaired lipid metabolism in aged mice as revealed by fasting-induced expression of apolipoprotein mRNAs in the liver and changes in serum lipids. Gerontology 50: 206-215, 2004.

30. Qin X and Tso P: The role of apolipoprotein AIV on the control of food intake. Curr Drug Targets 6: 145-151, 2005.

31. Whited KL, Lu D, Tso P, Kent Lloyd KC and Raybould HE: Apolipoprotein A-IV is involved in detection of lipid in the rat intestine. J Physiol 569: 949-958, 2005.

32. Culnan DM, Cooney RN, Stanley B and Lynch CJ: Apolipoprotein A-IV, a putative satiety/antiatherogenic factor, rises after gastric bypass. Obesity 17: 46-52, 2009.

33. Ostos MA, Conconi M, Vergnes L, Baroukh N, Ribalta J, Girona J, Caillaud JM, Ochoa A and Zakin MM: Antioxidative and antiatherosclerotic effects of human apolipoprotein A-IV in apolipoprotein E-deficient mice. Arterioscler Thromb Vasc Biol 21: 1023-1028, 2001

34. Broedl UC, Schachinger V, Lingenhel A, Lehrke M Stark R, Seibold F, Göke B, Kronenberg F, Parhofer KG and Konrad-Zerna A: Apolipoprotein A-IV is an independent predictor of disease activity in patients with inflammatory bowel disease. Inflamm Bowel Dis 13: 391-397, 2007.

35. Remaley AT, Stonik JA, Demosky SJ, Neufeld EB, Bocharov AV Vishnyakova TG, Eggerman TL, Patterson AP, Duverger NJ, Santamarina-Fojo S, et al: Apolipoprotein specificity for lipid efflux by the human ABCAI transporter. Biochem Biophys Res Commun 280: 818-823, 2001.

36. Stylianou IM, Affourtit JP, Shockley KR, Wilpan RY, Abdi FA, Bhardwaj S, Rollins J, Churchill GA and Paigen B: Applying gene expression, proteomics and single-nucleotide polymorphism analysis for complex trait gene identification. Genetics 178 1795-1805, 2008.
37. Jin EH, Shim SC, Kim HG, Chae SC and Chung HT: Polymorphisms of COTL1 gene identified by proteomic approach and their association with autoimmune disorders. Exp Mol Med 41: 354-361, 2009.

38. Weinberg RB: Apolipoprotein A-IV polymorphisms and diet-gene interactions. Curr Opin Lipidol 13: 125-134, 2002.

39. Vincent S, Planells R, Defoort C, Bernard MC, Gerber M, Prudhomme J, Vague P and Lairon D: Genetic polymorphisms and lipoprotein responses to diets. Proc Nutr Soc 61: 427-434, 2002.

40. Bai H, Liu R, Liu Y, Saku K and Liu BW: Distribution and effect of apo A-IV genotype on plasma lipid and apolipoprotein levels in a Chinese population. Acta Cardiol 63: 315-322, 2008.

41. Heilbronn LK, Noakes M, Morris AM, Kind KL and Clifton PM: $360 \mathrm{His}$ polymorphism of the apolipoprotein A-IV gene and plasma lipid response to envergy restricted diets in overweight subjects. Atherosclerosis 150: 187-192, 2000.

42. Kretowski A, Hokanson JE, McFann K, Kinney GL, SnellBergeon JK, Maahs DM, Wadwa RP, Eckel RH, Ogden LG and Garg SK: The apolipoprotein A-IV Gln360His polymorphism predicts progression of coronary artery calcification in patients with type 1 diabetes. Diabetologia 49: 1946-1954, 2006.

43. Antoine HJ, Pall M, Trader BC, Chen YD, Azziz R and Goodarzi MO: Genetic variants in peroxisome proliferatoractivated receptor gamma influence insulin resistance and testosterone levels in normal women, but not those with polycystic ovary syndrome. Fertil Steril 87: 862-869, 2007.

44. Codner E and Cassorla F: Puberty and ovarian function in girls with type 1 diabetes mellitus. Horm Res 71: 12-21, 2009.

45. Tsanadis G, Vartholomatos G, Korkontzelos I, Avgoustatos F, Kakosimos G, Sotiriadis A, Tatsioni A, Eleftheriou A and Lolis D: Polycystic ovarian syndrome and thrombophilia. Hum Reprod 17: 314-319, 2002.

46. Atiomo WU, Bates SA, Condon JE, Shaw S, West JH and Prentice AG: The plasminogen activator system in women with polycystic ovary syndrome. Fertil Steril 69: 236-241, 1998.

47. Dahlgren E, Janson PO, Johansson S, Lapidus L, Lindstedt G and Tengborn L: Hemostatic and metabolic variables in women with polycystic ovary syndrome. Fertil Steril 61: 455-460, 1994.

48. Kelly CJ, Lyall H, Petrie JR, Gould GW, Connell JM, Rumley A, Lowe GD and Sattar N: A specific elevation in tissue plasminogen activator antigen in women with polycystic ovarian syndrome. J Clin Endocrinol Metab 87: 3287-3290, 2002. 